\title{
Examen Nacional de Medicina (ENAM): Análisis de la última década de evaluaciones teóricas en los futuros médicos del Perú
}

\section{National Medicine Exam: an Analysis of the last decade of theoretical exams in peruvian last year medical students}

Correspondencia Giuston Mendoza Chuctaya giustonmch@gmail.com

\section{Recibido: 01/08/2021}

Arbitrado por pares

Aprobado: 23/09/2021

Citar como: Mendoza Chuctaya G, Calla Torres MR, Ramos Chuctaya KR, Mejía CR. Examen Nacional de Medicina (ENAM): Análisis de la última década de evaluaciones teóricas en los futuros médicos del Perú. Acta Med Peru. 2021:38(3):169-76. doi: https://doi. org/10.35663/amp.2021.383.2164

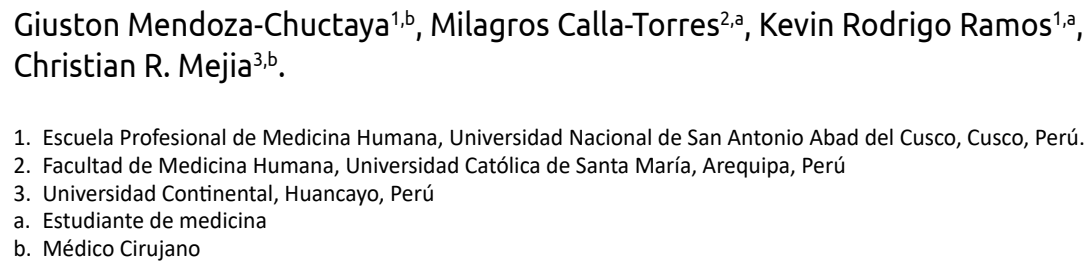

\section{RESUMEN}

Objetivos: Evaluar las notas del Examen Nacional de Medicina(ENAM) que sirvieron a médicos recién egresados para postular al servicio rural peruano entre 2009-2019. Materiales y Métodos: Estudio analítico, retrospectivo donde se realizó un análisis secundario de los registros a las convocatorias del Servicio Rural Urbano Marginal en Salud en Perú(SERUMS) entre los años 2009-2019. Se midió la frecuencia de los postulantes a plazas del SERUMS y la mediana de la nota del ENAM; cada uno de estos datos se presentó según distintas características, aplicando modelos bivariados y multivariados para obtener las razones de prevalencias. Resultados: Se evaluó a un total de 30750 médicos, donde 9087,17607 y 4056 fueron egresados de universidades públicas, privadas y del extranjero, respectivamente. El $43 \%$ del total de médicos inscritos al SERUMS desaprobaron el ENAM. Los porcentajes de desaprobados según universidades públicas, privadas y del extranjero fue $31 \%, 34 \%$ y $79 \%$ respectivamente. En el análisis multivariado se encontró que desaprobaban en mayor frecuencia los que eran de universidades privadas (RPa:1,42;IC95\%:1,37-1,47; valor $p<0,001$ ), los que estudiaron en una universidad de Lima Metropolitana (RPa:1,18;IC95\%:1,14-1,22;valor $p<0,001$ ), y los que egresaban de universidades del extranjero. Conclusiones: Se evidenció un aumento de médicos que realizan el SERUMS, donde un gran porcentaje desaprobaban el ENAM, especialmente aquellos médicos egresados de universidades privadas que se encontraban en Lima Metropolitana y de universidades del extranjero; resultados que sugieren que un gran porcentaje de médicos que hacen el servicio rural no están debidamente capacitados, al menos en el aspecto teórico que es lo que mide el ENAM.

Palabras clave: Evaluación Educacional; Educación de Pregrado en Medicina; Perú. 


\begin{abstract}
Objectives: To assess grades from the National Medical Exam (ENAM, according to its Spanish initials) that serve to last year medical students as a prerequisite to apply for places in Peruvian Medical Rural Service (SERUMS, according to its Spanish initials) between 2009 and 2019. Materials and Methods: This is an analytical retrospective study where a secondary analysis of registries for applying to the Peruvian Medical Rural Service (SERUMS) between years 2009 and 2019. Number of applicants to SERUMS places were determined, together with median values of ENAM results. Each of these pieces of information were presented according to different characteristics. Bivariate and multivariate models were used for obtaining prevalence rates. Results: More than thirty thousand students were assessed (30750), and 9087, 17607, and 4056 were from public, private, and foreign medical schools, respectively. Forty-three percent of those who registered tor applying the SERUMS failed the exam. Percentages of those who failed were $31 \%, 34 \%$, and $79 \%$ of those coming from public, private, and foreign medical schools, respectively. Multivariate analyses showed that those who most frequently failed came from private medical schools (odds ratio [OR]: $1.42 ; 95 \% \mathrm{Cl}: 1.37-1.47, \mathrm{p}<0.001$ ), those who studied medicine in a Lima City medical school (OR: 1.18; $95 \% \mathrm{Cl}: 1.14-1.22, \mathrm{p}<0.001)$, and those who studied outside Peru. Conclusions: There is a greater number of physicians doing rural service (SERUMS), but many of them fail the examination (ENAM), particularly those coming from private medical schools in Lima City and those who come from foreign medical schools. These results suggest that a great proportion of physicians applying for the rural service are not adequately qualified, at least in the theoretical areas which are measured by the ENAM.
\end{abstract}

Key words: Educational assessment, Pregraduate medical education, Peru

\section{INTRODUCCIÓN}

La educación médica ha evolucionado con los años, pasando a ser más activa, dinámica y reflexiva ${ }^{[1]}$, orientando su desarrollo a un enfoque por competencias (evaluación teórico-práctico), siendo el Examen Clínico Objetivo Estructurado (ECOE) la manera que ha demostrado una mejor validez y confiabilidad ${ }^{[2]}$. Distintos países han optado este enfoque como una estrategia para poder ejercer la medicina, como son el Examen de Licencia Médica en Estados Unidos ${ }^{[3]}$, el Examen Único Nacional de Conocimientos de Medicina Chile ${ }^{[4]}$, el Examen General al Egreso de la Licenciatura en Medicina General en México ${ }^{[5]}$, entre otros.

En el Perú, se instauró en el año 2003 el Examen Nacional de Medicina (ENAM), para poder medir la capacidad académica de los estudiantes que cursan el último año de la carrera ${ }^{[6]}$. Se trata de una prueba escrita de 200 preguntas de opción múltiple, que abarca las áreas de Medicina(30\%), Cirugía(15\%), Pediatría(18\%), Ginecología y Obstetricia(17\%), Salud Pública y Gestión(10\%) y Ciencias Básicas $(10 \%)^{[7]}$. Con los años este examen ha cobrado mayor importancia, pues representa un $70 \%$ de la nota final para adjudicar una plaza al Servicio Rural Urbano Marginal en Salud (SERUMS $)^{[8]}$, además, de ser un porcentaje para la adjudicación de una plaza en la especialidad médica en Perú ${ }^{[9]}$ y es un referente para el ranking de universidades y licenciamiento de las escuelas de medicina. ${ }^{[10,11]}$

Para el año 2016, el Ministerio de Salud peruano modificó el reglamento para hacer el SERUMS, indicando que sea requisito aprobar el ENAM ${ }^{[12]}$, siendo esta medida suspendida en el año 2018 -aduciendo tener insuficientes recursos humanos para mantener la capacidad resolutiva de los establecimientos de salud del país. ${ }^{[13]}$ Sin embargo, en el año 2019 el Colegio Médico del Perú estableció la obligatoriedad de aprobar el ENAM para poder adquirir la colegiatura, requisito obligatorio para ejercer profesionalmente la medicina. ${ }^{[14]}$ Por lo expuesto, nuestro objetivo fue evaluar las notas del ENAM que sirvieron a médicos recién egresados para postular al servicio rural peruano entre 2009-2019.

\section{MATERIAL Y MÉTODOS}

Estudio observacional de tipo transversal analítico, que se basó en data generada entre los años 2009-2019. La muestra fue el total de la población, conformada por el $100 \%$ de médicos inscritos a procesos de adjudicación del Servicio Rural Urbano Marginal de Salud (SERUMS), data que contiene la nota ENAM de todos los inscritos, promedio ponderado promocional y universidad de procedencia; esta información se encuentra de manera pública en la página web del Ministerio de Salud del Perú. El estudio incluyó los registros entre los años 2009-2019 (salvo del año 2016, por no encontrarse disponibles de forma online) y a las universidades peruanas que hayan tenido por lo menos alguna promoción participe en el proceso SERUMS, excluyéndose los datos del proceso de adjudicación equivalente.

Se diseñó una base de datos general en el programa Microsoft Exce ${ }^{\circledR}$ (versión para Windows 2013) con las siguientes variables: cantidad de inscritos al proceso SERUMS, universidad de procedencia (nacional o extranjera), tipo de universidad según su financiamiento (públicas o privadas), la variable ciudad de procedencia se dicotomizo en Lima Metropolitana (capital del Perú que alberga a la mayor cantidad de universidades del país) o no, regiones geográficas (costa, sierra, selva) y nota que obtuvo (en la escala vigesimal 0 a 20) en el Examen Nacional de Medicina que se clasificó como aprobados ( $\geq 11$ ) y desaprobados $(<11)$. 
Se preservó el anonimato de los participantes, así se aseguró la confidencialidad de la información obtenida y cumplió con los procesos éticos en investigación. La base de datos que fue revisada por los autores realizando un filtrado y control de calidad.

Se utilizó el programa estadístico Stata versión 11,1 (StataCorp LP, College Station, TX, USA). Para el análisis descriptivo se midió la frecuencia de los postulantes a plazas del SERUMS, la mediana de la nota del ENAM y cada uno de estos datos se presentó según el año de inscripción y procedencia de la universidad de egreso. Además, se obtuvo modelos bivariados y multivariados utilizando modelos lineales generalizados (con la familia Poisson, función de enlace log y ajuste para varianzas robustas); con esto se obtuvo las razones de prevalencias, los intervalos de confianza al 95\% y los valores $p$. Se consideró todo valor $p$ menor a 0,05 como estadísticamente significativo.

\section{RESULTADOS}

Durante los años 2009 al 2019, hubo un total de 30750 médicos inscritos para la adjudicación de una plaza SERUMS, egresados de 36 universidades peruanas (17 públicas y 19 privadas), 9087 inscritos fueron egresados de universidades públicas, 17607 egresados de universidades privadas y 4056 egresados de universidades del extranjero. El porcentaje de la tasa media de crecimiento anual fue de $5 \%$, siendo $10,1 \%$ el mayor aumento por año en egresados de universidades del extranjero, como se muestra en la Tabla 1.

Tabla 1. Total de médicos que adjudicaron al SERUMS por año y procedencia de universidad.

\begin{tabular}{ccccc}
\hline Año* & \multicolumn{2}{c}{ Univ. Nacionales } & $\begin{array}{c}\text { Univ. } \\
\text { Extranjeras }\end{array}$ & Total \\
\cline { 2 - 3 } & Publicas & Privadas & \\
\hline $\mathbf{2 0 0 9}$ & 929 & 1332 & 137 & 2398 \\
$\mathbf{2 0 1 0}$ & 796 & 1285 & 122 & 2203 \\
$\mathbf{2 0 1 1}$ & 878 & 1551 & 236 & 2665 \\
$\mathbf{2 0 1 2}$ & 362 & 1157 & 542 & 2061 \\
$\mathbf{2 0 1 3}$ & 907 & 1570 & 464 & 2941 \\
$\mathbf{2 0 1 4}$ & 1011 & 1979 & 706 & 3696 \\
$\mathbf{2 0 1 5}$ & 996 & 1937 & 648 & 3581 \\
$\mathbf{2 0 1 7}$ & 1074 & 2320 & 524 & 3918 \\
\hline $\mathbf{2 0 1 8}$ & 1035 & 2179 & 351 & 3565 \\
\hline $\mathbf{2 0 1 9}$ & 1099 & 2297 & 326 & 3722 \\
\hline Total & 9087 & 17607 & 4056 & 30750 \\
\hline \%TMCA & $1,8 \%$ & $6,2 \%$ & $10,1 \%$ & $5,0 \%$ \\
\hline
\end{tabular}

* Se excluyendo los registros del año 2016 por no estar disponible en la Web. \%TMCA: porcentaje de la tasa media de crecimiento anual.
En la Tabla 2 se muestra la mediana de las notas clasificados por años y por procedencia de universidad, donde se observa de manera global que la nota ENAM fue de 11,3; además, se muestra las notas promedio de egresados de universidades públicas, privadas y del extranjero. El $42,8 \%$ del total desaprobaron el ENAM; según la universidad de origen, el 31,3\% de los egresados de universidades públicas, el 33,9\% de egresados de universidades privadas y el 79,0\% egresados de universidades del extranjero habían desaprobado; lo cual se especifica en la Tabla 3.

En el análisis multivariado, se encontró que desaprobaban en mayor frecuencia los que eran de universidades privadas (RPa: 1,42; IC95\%: 1,37-1,47; valor $p<0,001$ ), los que estudiaron en una universidad de Lima Metropolitana (RPa: 1,18; IC95\%: 1,14-1,22; valor $\mathrm{p}<0,001$ ), en Bolivia (RPa: 1,66; IC95\%: 1,61-1,71; valor $p<0,001$ ), en Cuba (RPa: 8,45; IC95\%: 7,93-8,99; valor $p<0,001$ ), en Venezuela (RPa: 2,26; IC95\%: 1,93-2,65; valor $p<0,001$ ), en otros países de Latinoamérica (RPa: 1,59; IC95\%: 1,18-2,13; valor $\mathrm{p}=0,003$ ) y en otros lugares del mundo (RPa: 2,$67 ; \mathrm{IC} 95: \%$ 2,47-2,89; valor $p<0,001)$; en cambio, desaprobaron menos los de universidades de la serranía peruana (RPa: 0,83; IC95:\% 0,800,87 ; valor $p<0,001$ ); todas estuvieron ajustadas por el año en el que dio el ENAM y la nota del promedio ponderado promocional. Estos resultados se muestran en la Tabla 4.

En la figura 1.A se muestra una gráfica de cajas y bigotes de los promedios de notas ENAM obtenidas en periodo de estudio y en la figura 1.B el porcentaje de desaprobados en el ENAM por años.

\section{DISCUSIÓN}

La tendencia de médicos inscritos en los procesos SERUMS se ha incrementado a través de los años, al igual que la cantidad de médicos colegiados ${ }^{[15]}$, donde la mayor tasa de crecimiento anual fue de egresados de universidades del extranjero que tuvo el punto más alto el año 2014 y que a la fecha hay una tendencia a disminuir, esto probablemente debido a que cada vez es menor la cantidad de peruanos que migran al extranjero para cursar estudios de pregrado en medicina humana para luego retornar al país ${ }^{[16]}$, ya que hay una mayor oferta de facultades de medicina en el Perú y algunas han facilitado el ingreso y aumentado sus plazas. Esta situación pone en manifiesto el incremento acelerado de escuelas de medicina que ha pasado de 24 en 1998 a ser 52 al año 2016, principalmente en las universidades privadas. ${ }^{[17]}$ Dado que en el Perú existe una escasez significativa de recursos humanos en salud ${ }^{[18]}$, este crecimiento acelerado podría copar la demanda en menor tiempo, sin embargo, se requiere no solo cantidad sino también profesionales competentes. Teniendo en cuenta el enfoque de educación de calidad, el Ministerio de Educación junto con la Superintendencia Nacional de Educación Superior Universitaria (SUNEDU) a través de la Ley Universitaria $\mathrm{N}^{\circ} 30220^{[19]}$, han instaurado filtros para la mejora de la calidad educativa gracias al licenciamiento de universidades y de los programas de pregrado. ${ }^{[10.11]}$ 
Tabla 2. Medianas de calificación obtenida en el Examen Nacional de Medicina (ENAM) según año y procedencia de universidad.

\begin{tabular}{|c|c|c|c|c|c|c|c|c|c|c|c|}
\hline Año* & 2009 & 2010 & 2011 & 2012 & 2013 & 2014 & 2015 & 2017 & 2018 & 2019 & Total \\
\hline \multicolumn{12}{|l|}{ Univ. Peruanas Públicas } \\
\hline U. Nac Mayor de San M. & 13,8 & 13,9 & 13,3 & 13,5 & 13,7 & 12,6 & 13,3 & 15,1 & 13,8 & 14,6 & 13,8 \\
\hline U. Nac de Piura & 12,3 & 13,2 & 13,6 & 13,3 & 13,8 & 12,1 & 13,2 & 13,3 & 11,6 & 12,6 & 12,8 \\
\hline U. Nac Pedro Ruiz Gallo & 13,3 & 12,9 & 12,0 & 10,6 & 12,9 & 12,0 & 11,3 & 13,7 & 13,2 & 13,3 & 12,7 \\
\hline U. Nac Hermilio Valdizan & 12,7 & 13,0 & 12,4 & 12,6 & - & 11,8 & 12,2 & 12,8 & 12,5 & 12,1 & 12,4 \\
\hline U. Nac de Trujillo & 12,9 & 12,6 & 11,5 & 11,4 & 12,1 & 11,1 & 10,9 & 13,4 & 13,3 & 13,7 & 12,2 \\
\hline U. Nac San Antonio Abad & 12,9 & 12,4 & 11,7 & 11,4 & 12,3 & 11,3 & 11,5 & 13,6 & 12,6 & 13,0 & 12,2 \\
\hline U. Nac San Agustín & 13,3 & 13,1 & 12,3 & 11,6 & 12,7 & 10,7 & 10,8 & 13,6 & 12,0 & 12,7 & 12,1 \\
\hline U. Nac de San Martín $\ddagger$ & - & - & - & - & - & - & - & 12,0 & 11,7 & 12,3 & 12,0 \\
\hline U. Nac Jorge Basadre G. & 13,1 & 13,5 & 11,9 & 10,5 & 12,5 & 11,0 & 11,3 & 13,6 & 11,9 & 12,3 & 12,0 \\
\hline U. Nac del Centro del Perú & 11,9 & 11,9 & 11,3 & 10,6 & 12,3 & 11,2 & 11,9 & 12,8 & 11,5 & 12,7 & 12,0 \\
\hline U. Nac Jose Faustino S. & 12,0 & 11,7 & 11,6 & 11,3 & 11,4 & 11,0 & 11,4 & 13,5 & 12,1 & 12,3 & 11,8 \\
\hline U. Nac de Cajamarca & 11,9 & 11,8 & 11,2 & $(, /)$, & 11,3 & 10,7 & 10,9 & 13,3 & 11,8 & 12,9 & 11,8 \\
\hline U. Nac Federico Villarreal & 12,1 & 12,4 & 11,8 & 12,0 & 11,6 & 10,2 & 10,7 & 12,0 & 11,1 & 11,2 & 11,5 \\
\hline U. Nac del Altiplano & 10,2 & 10,5 & 11,0 & 10,7 & 11,8 & 10,4 & 10,8 & 12,9 & 11,2 & 11,8 & 11,1 \\
\hline U. Nac de Ucayali & 12,1 & 12,8 & 10,9 & - & 10,9 & 8,5 & 11,5 & 12,0 & 11,1 & 10,9 & 11,1 \\
\hline U. Nac San Luis Gonzaga & 11,2 & 11,1 & 10,3 & - & 10,7 & 9,2 & 11,2 & 12,3 & 10,8 & 11,1 & 11,0 \\
\hline U. Nac de la Amazonía P. & 10,9 & 10,6 & 10,6 & 10,8 & 10,8 & 9,9 & 9,7 & 11,1 & 11,9 & 11,5 & 10,7 \\
\hline Total & 12,4 & 12,6 & 11,9 & 12,1 & 12,3 & 11,0 & 11,3 & 13,2 & 12,2 & 12,5 & 12,1 \\
\hline \multicolumn{12}{|l|}{ Univ. Peruanas Privadas } \\
\hline U. Peruana de Ciencias A. $¥$ & - & - & - & - & - & 11,6 & 13,4 & 14,2 & 14,1 & 14,6 & 13,8 \\
\hline U. Peruana Cayetano H. & 13,2 & 13,3 & 14,8 & 13,9 & 13,5 & 13,0 & 13,6 & 14,8 & 13,6 & 14,0 & 13,7 \\
\hline U. Católica Santo Toribioł & - & - & - & - & 13,7 & 13,2 & 12,2 & 14,0 & 12,8 & 13,5 & 13,3 \\
\hline U. Continentalł & - & - & - & - & - & - & - & - & - & 13,1 & 13,1 \\
\hline U. Peruana Unión‡ & - & - & - & - & - & - & - & - & - & 13,1 & 13,1 \\
\hline U. Andina del Cuscoł & - & - & - & - & - & - & - & 14,8 & 12,2 & 12,9 & 12,9 \\
\hline U. Científica del Sur & 12,7 & 12,5 & 11,7 & 12,0 & 12,3 & 11,4 & 11,7 & 13,2 & 12,1 & 12,5 & 12,2 \\
\hline U. San Martin de Porres & 12,3 & 12,4 & 11,7 & 12,1 & 12,6 & 11,1 & 12,0 & 13,3 & 11,8 & 12,4 & 12,1 \\
\hline U. Católica Santa Maria & 12,3 & 11,9 & 10,8 & 9,8 & 11,8 & 10,5 & 10,8 & 12,9 & 11,6 & 12,0 & 11,6 \\
\hline U. Andina Néstor Caceresł & - & - & - & - & - & - & - & 12,0 & 11,5 & 11,2 & 11,4 \\
\hline U. Priv Antenor Orrego & 11,9 & 11,6 & 10,9 & 10,5 & 12,1 & 9,9 & 10,5 & 12,3 & 11,5 & 12,0 & 11,4 \\
\hline U. Ricardo Palma & 11,8 & 11,3 & 10,5 & 11,0 & 11,9 & 10,1 & 10,6 & 12,6 & 11,6 & 12,5 & 11,3 \\
\hline U. Alas Peruanas $\ddagger$ & - & - & - & - & - & - & - & - & 10,8 & 11,3 & 11,2 \\
\hline U. Priv de Tacna & 11,5 & 11,2 & 10,3 & 8,9 & 11,0 & 9,4 & 10,2 & 12,0 & 11,2 & 11,7 & 10,8 \\
\hline U. Peruana Los Andes & 10,8 & 10,6 & 9,3 & 9,3 & 11,1 & 9,7 & 10,2 & 11,2 & 10,0 & 10,9 & 10,4 \\
\hline U. San Pedro & 11,7 & 10,9 & 9,9 & 10,0 & 10,4 & 8,7 & 7,6 & 11,5 & 10,8 & 11,5 & 10,3 \\
\hline U. Priv San Juan Bautista & 10,7 & 10,6 & 9,5 & 10,1 & 10,1 & 10,0 & 9,9 & 9,8 & 11,4 & 10,3 & 10,2 \\
\hline U. de Chiclayo & 10,6 & 10,3 & 10,1 & 9,6 & 10,5 & 9,1 & 9,2 & 11,8 & 10,3 & 11,1 & 10,1 \\
\hline U. Cesar Vallejo & 11,1 & 11,2 & 9,6 & 9,5 & - & 8,9 & 9,4 & 11,0 & 10,1 & 9,8 & 10,1 \\
\hline Total & 11,9 & 11,6 & 10,7 & 11,2 & 11,8 & 10,3 & 10,8 & 12,3 & 11,6 & 11,8 & 11,4 \\
\hline Univ. Extranjeras & 0,0 & 10,2 & 9,5 & 9,7 & 9,4 & 9,0 & 8,8 & 9,5 & 10,0 & 11,1 & 9,4 \\
\hline Total & 12,0 & 11,8 & 11,0 & 10,9 & 11,6 & 10,2 & 10,6 & 12,2 & 11,6 & 12,0 & 11,3 \\
\hline
\end{tabular}

*Se excluyendo los registros del año 2016 por no estar disponible en la Web. ‡ Universidades que tuvieron su primera promoción después del año 2009. 
Tabla 3. Porcentaje (\%) de reprobados en el Examen Nacional de Medicina (ENAM) según año y procedencia de universidad.

\begin{tabular}{|c|c|c|c|c|c|c|c|c|c|c|c|}
\hline Año* & 2009 & 2010 & 2011 & 2012 & 2013 & 2014 & 2015 & 2017 & 2018 & 2019 & total \\
\hline \multicolumn{12}{|l|}{ Univ. Peruanas Públicas } \\
\hline U. Nac Mayor de San M. & 5,6 & 3,8 & 8,6 & 10,6 & 4,2 & 18,8 & 9,2 & 2,7 & 0,0 & 3,5 & 6,6 \\
\hline U. Nac de Piura & 24,5 & 5,7 & 11,4 & 33,3 & 3,3 & 29,4 & 15,4 & 6,8 & 23,8 & 16,1 & 16,8 \\
\hline U. Nac Pedro Ruiz Gallo & 11,1 & 9,1 & 23,5 & 50,0 & 13,8 & 32,8 & 42,3 & 4,2 & 16,9 & 9,9 & 18,7 \\
\hline U. Nac San Antonio Abad & 14,0 & 26,2 & 30,5 & 20,0 & 16,3 & 35,7 & 28,9 & 2,8 & 18,8 & 16,9 & 21,1 \\
\hline U. Nac Hermilio Valdizan & 0,0 & 20,0 & 16,7 & 0,0 & 0,0 & 37,5 & 20,5 & 6,8 & 29,4 & 39,3 & 21,4 \\
\hline U. Nac de Trujillo & 17,0 & 11,8 & 30,7 & 23,3 & 14,7 & 39,4 & 51,2 & 4,8 & 18,1 & 10,4 & 22,9 \\
\hline U. Nac de San Martín‡ & - & - & - & - & - & - & - & 30,0 & 29,7 & 18,8 & 27,7 \\
\hline U. Nac San Agustín & 11,8 & 18,8 & 28,0 & 44,4 & 17,0 & 56,3 & 56,5 & 9,0 & 25,7 & 11,5 & 29,5 \\
\hline U. Nac Jose Faustino S. & 18,9 & 38,8 & 29,6 & 35,3 & 36,5 & 50,0 & 32,4 & 2,8 & 30,8 & 14,7 & 29,6 \\
\hline U. Nac del Centro del Perú & 32,4 & 29,1 & 44,2 & 66,7 & 32,6 & 43,3 & 27,8 & 9,4 & 40,3 & 14,0 & 31,3 \\
\hline U. Nac Jorge Basadre G. & 18,8 & 18,2 & 38,2 & 62,5 & 26,3 & 46,9 & 41,7 & 20,7 & 25,0 & 20,7 & 31,9 \\
\hline U. Nac de Cajamarca & 20,0 & 34,6 & 45,5 & - & 48,0 & 58,1 & 53,1 & 20,0 & 33,3 & 11,6 & 33,3 \\
\hline U. Nac Federico Villarreal & 32,0 & 27,8 & 35,9 & 28,8 & 37,9 & 66,4 & 58,0 & 34,6 & 46,8 & 46,4 & 41,6 \\
\hline U. Nac del Altiplano & 60,6 & 56,3 & 46,2 & 57,1 & 29,2 & 64,6 & 54,1 & 29,7 & 37,7 & 28,6 & 46,7 \\
\hline U. Nac de Ucayali & 21,4 & 0,0 & 50,0 & - & 57,1 & 84,0 & 36,4 & 18,2 & 48,1 & 55,2 & 47,5 \\
\hline U. Nac San Luis Gonzaga & 45,0 & 44,4 & 58,5 & - & 52,9 & 76,6 & 49,1 & 31,1 & 52,7 & 47,2 & 49,7 \\
\hline U. Nac de la Amazonía P. & 52,0 & 53,3 & 66,7 & 60,0 & 55,1 & 66,7 & 83,3 & 44,0 & 23,7 & 35,3 & 55,1 \\
\hline Total & 24,0 & 24,9 & 35,3 & 37,8 & 27,8 & 50,4 & 41,2 & 16,3 & 29,5 & 23,5 & 31,3 \\
\hline \multicolumn{12}{|l|}{ Univ. Peruanas Privadas } \\
\hline U. Católica Santo Toribioł & - & - & - & - & 0,0 & 0,0 & 0,0 & 0,0 & 8,3 & 0,0 & 1,5 \\
\hline U. Peruana Unión & - & - & - & - & - & - & - & - & - & 3,7 & 3,7 \\
\hline U. Peruana de Ciencias A.‡ & & - & - & - & - & 3,2 & 5,4 & 2,6 & 6,9 & 4,2 & 4,6 \\
\hline U. Peruana Cayetano H. & 12,4 & 7,0 & 10,0 & 1,4 & 5,0 & 7,8 & 1,1 & 1,8 & 6,1 & 1,9 & 5,6 \\
\hline U. Andina del Cusco $¥$ & - & - & - & - & - & - & - & 0,0 & 10,9 & 9,7 & 8,3 \\
\hline U. Continental‡ & - & - & - & - & - & - & - & - & - & 8,3 & 8,3 \\
\hline U. Científica del Sur & 15,2 & 12,5 & 26,1 & 16,2 & 21,3 & 44,4 & 27,6 & 10,6 & 25,0 & 15,3 & 21,5 \\
\hline U. San Martin de Porres & 22,1 & 21,7 & 37,4 & 22,9 & 15,8 & 46,3 & 28,9 & 8,5 & 27,8 & 19,4 & 24,7 \\
\hline U. Andina Néstor Cáceresł & - & - & - & - & - & - & - & 28,0 & 18,2 & 40,5 & 32,9 \\
\hline U. Alas Peruanas & - & - & - & - & - & - & - & - & 50,0 & 35,3 & 37,5 \\
\hline U. Católica Santa María & 23,1 & 28,5 & 51,2 & 70,8 & 33,1 & 64,1 & 52,9 & 13,0 & 34,3 & 28,2 & 37,8 \\
\hline U. Ricardo Palma & 26,6 & 40,6 & 58,3 & 48,1 & 23,4 & 73,0 & 58,2 & 17,9 & 34,5 & 16,7 & 42,1 \\
\hline U. Priv Antenor Orrego & 36,7 & 32,3 & 51,0 & 57,9 & 25,3 & 77,2 & 59,4 & 23,7 & 36,8 & 29,1 & 42,2 \\
\hline U. Priv de Tacna & 29,7 & 43,9 & 69,7 & 91,7 & 48,6 & 79,7 & 62,7 & 27,0 & 28,6 & 32,7 & 52,8 \\
\hline U. Peruana Los Andes & 53,4 & 59,6 & 81,9 & 93,3 & 43,9 & 90,7 & 61,6 & 39,3 & 71,6 & 50,0 & 61,2 \\
\hline U. de Chiclayo & 56,3 & 63,6 & 66,7 & 85,7 & 58,1 & 95,8 & 88,1 & 38,9 & 62,7 & 45,5 & 62,1 \\
\hline U. Priv San Juan Bautista & 53,2 & 61,9 & 76,1 & 73,4 & 65,9 & 73,1 & 68,8 & 64,8 & 43,0 & 58,8 & 62,9 \\
\hline U. San Pedro & 34,8 & 50,0 & 73,2 & 70,1 & 61,9 & 88,3 & 100 & 40,0 & 56,6 & 38,2 & 63,6 \\
\hline U. Cesar Vallejo & 40,6 & 42,0 & 72,3 & 91,3 & - & 88,4 & 86,3 & 49,1 & 69,3 & 75,2 & 70,5 \\
\hline Total & 33,7 & 38,6 & 56,2 & 60,2 & 33,5 & 59,4 & 50,1 & 22,8 & 34,7 & 27,0 & 33,9 \\
\hline Univ. Extranjeras & 82,9 & 66,4 & 81,8 & 79,3 & 84,9 & 86,1 & 87,5 & 80,0 & 69,8 & 47,2 & 79,0 \\
\hline Total & 30,9 & 32,5 & 49,5 & 51,4 & 39,0 & 64,8 & 56,6 & 31,0 & 37,8 & 32,3 & 42,8 \\
\hline
\end{tabular}

*Se excluyendo los registros del año 2016 por no estar disponible en la Web. ¥ Universidades que tuvieron su primera promoción después des año 2009. 
Tabla 4. Análisis bivariado y multivariado de las características de las universidades asociadas al aprobar el examen del ENAM, 2009-2019.

\begin{tabular}{|c|c|c|c|c|}
\hline \multirow{2}{*}{ Variables } & \multicolumn{2}{|c|}{ Desaprobó } & \multirow{2}{*}{ Bivariado } & \multirow{2}{*}{ Multivariado } \\
\hline & No & Si & & \\
\hline \multicolumn{5}{|l|}{ Tipo de universidad* } \\
\hline Pública/nacional & $6445(70,9 \%)$ & $2642(29,1 \%)$ & Cat. de comparación & Cat. de comparación \\
\hline Privada/particular & $10282(58,4 \%)$ & $7325(41,6 \%)$ & $1,44(1,38-1,48)<0,001$ & $1,42(1,37-1,47)<0,001$ \\
\hline \multicolumn{5}{|l|}{ Lima metropolitana* } \\
\hline No & $8308(58,1 \%)$ & $5981(41,9 \%)$ & Cat. de comparación & Cat. de comparación \\
\hline $\mathrm{Si}$ & $8419(67,9 \%)$ & $3986(32,1 \%)$ & $0,77(0,74-0,79)<0,001$ & $1,18(1,14-1,22)<0,001$ \\
\hline \multicolumn{5}{|l|}{ Región peruana* } \\
\hline Costa & $13112(62,9 \%)$ & $7746(37,1 \%)$ & Cat. de comparación & Cat. de comparación \\
\hline Sierra & $3293(63,3 \%)$ & $1911(36,7 \%)$ & $0,99(0,95-1,03) 0,580$ & $0,83(0,80-0,87)<0,001$ \\
\hline Selva & $322(51,0 \%)$ & $310(49,0 \%)$ & $1,32(1,22-1,43)<0,001$ & $1,00(0,91-1,09) 0,942$ \\
\hline \multicolumn{5}{|l|}{ País donde estudió** } \\
\hline Perú & $16725(62,7 \%)$ & $9963(37,3 \%)$ & Cat. de comparación & Cat. de comparación \\
\hline Bolivia & $357(17,8 \%)$ & $1651(82,2 \%)$ & $2,20(2,14-2,26)<0,001$ & $1,66(1,61-1,71)<0,001$ \\
\hline Cuba & $302(19,5 \%)$ & $1249(80,5 \%)$ & $2,16(2,10-2,22)<0,001$ & $8,45(7,93-8,99)<0,001$ \\
\hline Venezuela & $136(54,4 \%)$ & $114(45,6 \%)$ & $1,22(1,07-1,40) 0,004$ & $2,26(1,93-2,65)<0,001$ \\
\hline Otras de Latinoamérica & $52(30,4 \%)$ & $119(69,6 \%)$ & $1,86(1,67-2,06)<0,001$ & $1,59(1,18-2,13) 0,003$ \\
\hline Otras del mundo & $7(8,5 \%)$ & $75(91,5 \%)$ & $2,45(2,29-2,62)<0,001$ & $2,67(2,47-2,89)<0,001$ \\
\hline
\end{tabular}

Las razones de prevalencia (izquierda), intervalos de confianza al 95\% (dentro del paréntesis) y valores $p$ (derecha) fueron obtenidos con los modelos lineales generalizados (familia Poisson, función de enlace log y ajuste para varianzas robustas). Los modelos multivariados fueron ajustados por el año en el que dio el ENAM y la nota del promedio ponderado promocional. ${ }^{*} \mathrm{~N}=26694 .{ }^{* *} \mathrm{~N}=30750$

En general, 4 de cada 10 egresados desaprobaron el ENAM, estos resultados sugieren que un gran porcentaje de médicos aún no está capacitado para atender a poblaciones de zonas rurales del Perú, sin embargo, esta evaluación no mide en conjunto las habilidades en la práctica ni la formación académica -como lo hace el Examen Clínico Objetivo Estructurado ${ }^{[2]}$, lo que es una de las mayores críticas a este examen. Otro factor podría ser la

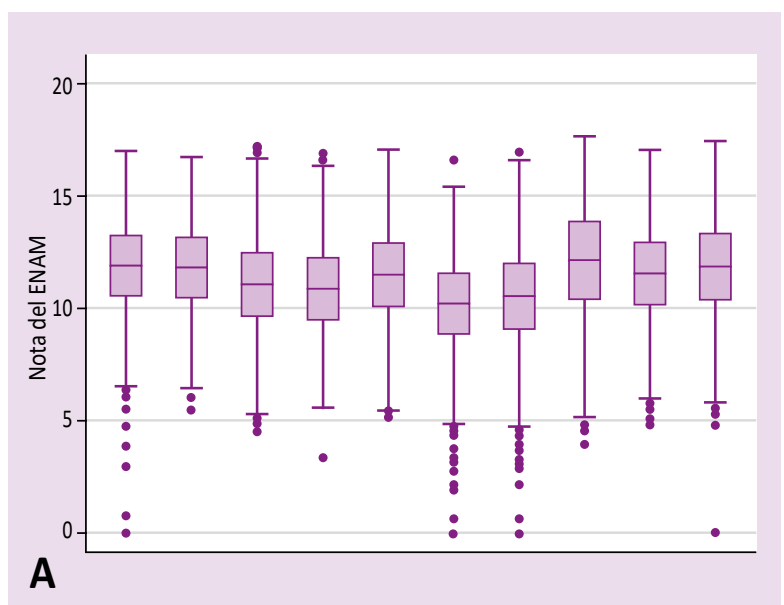

inadecuada formación académica por parte de las universidad, diferentes niveles de dificultad del examen con el paso de los años ${ }^{[20,21]}$ y el hecho que la mayoría de estudiantes que rinden este examen cursan el internado médico (último año de medicina), etapa donde se ha reportado falta de descansos postguardia ${ }^{[22]}$ y alta prevalencia de síndrome burnout ${ }^{[23]}$ que podría llevar a un deficiente desempeño profesional e influir

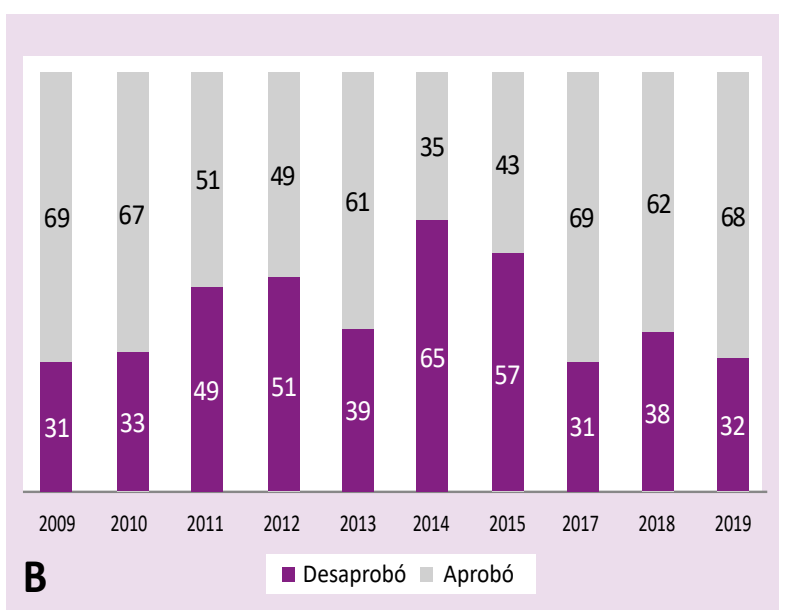

Figura 1. Gráfica de cajas y bigotes del promedio de notas ENAM (A) y del porcentaje global de aprobados/reprobados (B) según años. 
en una nota menor. Por tal razón, algunas universidades han implementado estrategias como convenios con academias de preparación médica, creación de cursos y evaluaciones semanales dirigidos a sus alumnos y docentes. ${ }^{[24,25]}$ Un ejemplo de ello, es el programa de la Universidad Peruana Cayetano Heredia que ha optado medir la competencia medica con estas estrategias, teniendo una respuesta positiva en sus estudiantes. ${ }^{[26]}$

Nuestros resultados concuerdan con otras investigaciones ${ }^{[16,27]}$ en donde la mediana de notas ENAM en egresados de universidades públicas se mantuvieron mayores en comparación de egresados de universidades privadas y extranjeras, aunque el motivo no es claro, algunos estudios refieren que posiblemente el entorno y los métodos de enseñanza en las escuelas privadas sean menos propicios que los de las escuelas públicas. ${ }^{[28]}$ Además, los promedios fueron aprobatorios en los egresados de universidades públicas y privadas en comparación de egresados de universidades del extranjero, probablemente debido a la distinta formación académica de cada universidad y país, ya que la enseñanza y evaluaciones están orientadas en el contexto epidemiológico propio del Perú.

El análisis multivariado muestra un menor porcentaje de desaprobados en aquellos médicos egresados de escuelas de medicina que no pertenecen a Lima Metropolitana, esto podría deberse a que Lima concentra la mayor cantidad de universidades privadas, ya que como se discutió anteriormente, los promedios de egresados de universidades privadas son más bajos respecto a las universidades públicas. Además, en cuanto a la distribución de regiones geográficas, se vio que ser egresado de una universidad ubicada en la sierra del Perú es un factor protector para no desaprobar el ENAM en comparación a ser egresado de una universidad de la costa; estos hallazgos concuerdan con el estudio de Hernández Gálvez et al. ${ }^{[29]}$ donde también se encontró diferencias significativas entre el puntaje de médicos según las distintas regiones geográficas, sin embargo, hacen falta más estudios para determinar los factores que contribuyen a estas diferencias.

A pesar de que este examen ha generado mucha controversia por su forma de medición, es actualmente el único indicador objetivo de la formación académica de los estudiantes de medicina del Perú. En el año 2019 se inició una prueba piloto para la aplicación del Examen Clínico Objetivo Estructurado (ECOE) a los estudiantes que cursaban el último año de medicina, sin embargo, los resultados no han sido publicados y aún no existe un reglamento que lo avale y garantice su continuidad.

La principal limitación del estudio fue el no tener los registros oficiales de las notas individuales del ENAM pues la Asociación Peruana de Facultades de Medicina (ASPEFAM), ente que se encarga de organizar este examen, no hace publica esta información, por ello, se utilizó los registros de los procesos SERUMS. La información en nuestro estudio no representa en su totalidad a los estudiantes que rindieron este examen en su primer intento, ya que, por distintos motivos un médico podría no haberse inscrito para la adjudicación de una plaza SERUMS en su año correspondiente. Además, este trabajo no pretende establecer un ranking de universidades ya que estas tienen distintos años en la que su primera promoción rindió el examen.

\section{CONCLUSIONES}

En conclusión, nuestro estudio evidenció un crecimiento acelerado del número de médicos que realizan el SERUMS, donde un gran porcentaje de estos desaprobaban el ENAM, especialmente aquellos egresados de universidades de privadas; que se encontraban en Lima Metropolitana y de médicos egresados de universidades del extranjero. Resultados que sugieren que un gran porcentaje de médicos que hacen el servicio rural no están debidamente capacitados, al menos en el aspecto teórico, que es lo que mide el ENAM. Por lo que se recomienda que se tome en cuenta la aplicación permanente del Examen Clínico Objetivo Estructurado para futuras promociones de médicos, ya que la nota aprobatoria del ENAM es un requisito para poder ejercer la medicina en el Perú.

\section{Agradecimientos:}

La presente investigación agradece a Claudia Guerra Rios, Gloria Lissett Bedia Serrano, Katherine Fernanda Casafranca Serrano, Esperanza Vizcarra Vargas quienes participaron activamente en la revisión de la versión final de manuscrito.

\section{Conflictos de Interés:}

Los autores declaramos no presentar ningún conflicto de interés

\section{Fuentes de financiamiento:}

Autofinanciado

\section{ORCID}

1. Giuston Mendoza Chuctaya: https://orcid.org/ 0000-00025389-5640

2. Milagros Rocio Calla Torres: https://orcid.org/ 0000-00030328-876X

5. Kevin Rodrigo Ramos Chuctaya: https://orcid.org/ 00000001-9183-6281

3. Christian Mejía Álvarez: https://orcid.org/ 0000-0002-59407281

\section{REFERENCIAS BIBLIOGRÁFICAS}

1. Peña LG, Dávalos ELG, Rodríguez PPF, Blanco ZE, Viera HRV, Rocha CIC. La evaluación clínica objetiva estructurada desde el área de formación médica general. Inv Educ Med. 2019; 8: 40-50. Disponible en: https://www.medigraphic.com/cgi-bin/new/resumen. cgi?IDARTICULO=93528

2. Khan KZ, Ramachandran S, Gaunt K, Pushkar P. The Objective Structured Clinical Examination (OSCE): AMEE Guide No. 81. Part I: An historical and theoretical perspective. Med Teach. 2013;35:e1437-46. doi: 10.3109/0142159X.2013.818634 
3. Haist SA, Katsufrakis PJ, Dillon GF. The evolution of the United States Medical Licensing Examination (USMLE). Enhancing assessment of practice-related competencies. JAMA. 2013;310(21):2245-6. doi: 10.1001/jama.2013.282328

4. Ministerio de Salud de Chile;Subsecretaría de Salud Pública. Ley 20.261.Crea examen único nacional de conocimientos de medicina, incorpora cargos que indica al sistema de alta dirección pública y modifica la ley $\mathrm{N}^{0} \mathbf{1 9} .664$. [consultado 22 de enero de 2020]. Disponible en: https://www.leychile.cl/Navegar?idNorma=270584/

5. Guerrero AJJ, Romero-González J, Díaz RJ, Díaz TB, Ríos RR, Zesati PGI. Evaluación de medicina en México. CULCyT.2015;12:238-43. [consultado 22 de enero de 2020]. Disponible en:http:// openjournal.uacj.mx/ojs/index.php/culcyt/article/view/764/731.

6. Torres-Noriega J. Los exámenes nacionales de medicina (ENAM) en el Perú. Rev Peru Med Exp Salud Publica. 2008;25(3):316-18. doi: 10.17843/rpmesp.2008.253.1289

7. Asociación Peruana de Facultades de Medicina. Examen nacional de medicina: Tabla de especificaciones. II Taller de análisis y revisión de la tabla de especificaciones del ENAM. Lima-Perú, 2008. [Internet]. Lima, Perú. [consultado 22 de enero de 2020]. Disponible en: http// www.aspefam.org.pe/enam/tabla.pdf.

8. Diario Oficial El Peruano. Decreto Supremo $N^{\circ}$ 007-2008-SA. Aprueban modificaciones al Reglamento de la Ley $N^{\circ} 23330$ Ley del servicio Rural y Urbano Marginal de Salud - SERUMS [fecha de acceso 22 de enero de 2020]. Disponible en: https://www.gob.pe/ institucion/minsa/normas-legales/248433-007-2008-sa/

9. Diario Oficial El Peruano. Decreto Supremo $\mathrm{N}^{\circ}$ 007-2017-SA. Aprueba el Reglamento de la Ley $N^{\circ} 30453$, Ley del Sistema Nacional de Residentado Médico (SINAREME). [fecha de acceso 22 de enero de 2020]. Disponible en: https://busquedas.elperuano. pe/normaslegales/decreto-supremo-que-aprueba-el-reglamentode-la-ley-n-30453-decreto-supremo-n-007-2017-sa-1492036-2/

10. Superintendencia Nacional de Educación Superior Universitaria. Modelo de licenciamiento de programa de pregrado de medicina. Lima: SUNEDU; 2018. Disponible en: https://www.sunedu.gob.pe/ licenciamiento-programas-medicina-humana/

11. Mayta-Tristán P, Toro-Huamanchumo CJ, Alhuay-Quispe J, PachecoMendoza J. Producción científica y licenciamiento de escuelas de medicina en el Perú. Rev Peru Med Exp Salud Publica. 2019;36:10615. Disponible en: https://rpmesp.ins.gob.pe/index.php/rpmesp/ article/view/4315/3250

12. Ministerio de Salud de Perú. Resolución Ministerial №785-2016MINSA: Modificar el literal g) del artículo 23 del Reglamento de la Ley $N^{\circ} 23330$, Ley del Servicio Rural y Urbano Marginal de SaludSERUMS, 5 de octubre de 2016[consultado 22 de enero de 2020]. Disponible en: https://www.gob.pe/institucion/minsa/normaslegales/191726-785-2016-minsa/

13. Diario Oficial El Peruano.Ministerio de Salud.Resolución Ministerial N²63-2018-MINSA:Suspenden durante el año 2018 el requisito señalado en el literal g)del artículo 23 del Reglamento de la Ley $\mathrm{N}^{\circ} 23330,28$ de marzo de 2018. [consultado 22 de enero de 2020]. Disponible en: https://busquedas.elperuano.pe/normaslegales/ suspenden-durante-el-ano-2018-el-requisito-senalado-en-el-liresolucion-ministerial-n-263-2018minsa-1632025-1/

14. Morán-ortiz J, Cabani-Ravello L. Aprobar el Examen Nacional de Medicina será obligatorio para ejercer como médico cirujano en el Perú. Acta Med Peru. 2019;36(1):3-4. doi: 10.35663/ amp.2019.361.683

15. Ichiro PC, Garcia-Solorzano F, Zafra-Tanaka JH, Huapaya-Huertas OS, Taype-Rondan A. Tendencias en el número de médicos titulados anualmente en el Perú, 2007-2016. Acta Med Peru. 2019;36:19-25. Disponible en: http://www.scielo.org.pe/scielo.php?script=sci_ arttext\&pid=S1728-59172019000100004
16. Mendoza-Chuctaya G, Mejía CR, Cano-Pucapuca J. Diferencias en la nota del ENAM y el promedio ponderado universitario en postulantes al servicio rural peruano, 2008-2015. Educ Med. 2018;20(S1):104-9. doi: 10.1016/j.edumed.2017.10.016

17. Mayta-Tristán $P$, Cuentas $M$, Núñez-Vergara $M$. Responsabilidad de las instituciones ante la proliferación de escuelas de medicina en el Perú. Acta Med Per. 2016;33(3):178-82. doi: 10.35663/ amp.2016.333.109

18. Jimenez MM, Bui AL, Mantilla E, Miranda JJ. Human resources for health in Peru: recent trends (2007-2013) in the labour market for physicians, nurses and midwives. Hum Resour Health. 2017;15(1):69. doi: 10.1186/s12960-017-0243-y

19. Congreso de la República de Perú. Ley N³0220: Ley Universitaria. Lima; 2014. [consultado 22 de enero de 2020]. Disponible en: http://www.minedu.gob.pe/reforma-universitaria/pdf/ley_ universitaria.pdf

20. Mendoza-Chuctaya G, Barreto JB, Agramonte-Vilca M, Ruiz-Esquivel J. Examen Nacional de Medicina del Perú: análisis y variación de resultados en una escuela de Medicina. 2008-2015. Educ Med [Internet]. 2018. doi: 10.1016/j.edumed.2018.10.002

21. Mejia CR, Ruiz-Urbina FN, Benites-Gamboa D, Albitres-Flores L, Mena LS, Fasanando-Vela R. Percepciones de utilidad y preparación para el Examen Nacional de Medicina en 10 facultades peruanas, 2017. Educ Med. 2019;20:124-30. doi: 10.1016/j. edumed.2017.10.021

22. Nieto-gutierrez W, Taype-rondan A, Bastidas F, Casiano-Celestino $\mathrm{R}$, Inga-berrospi $\mathrm{F}$. Percepción de médicos recién egresados sobre el internado médico en Lima, Perú 2014. Acta Med Perú. 2016;33(2):105-10. doi: 10.35663/amp.2016.332.59

23. Suca-Saavedra R, López-Huamanrayme E. Síndrome de burnout en internos de medicina del Perú: ¿es un problema muy prevalente? Rev Neuropsiquiatr. 2016;79:69-70. Disponible en: http://www.scielo.org.pe/scielo.php?script=sci arttext\&pid=S0034-85972016000100010

24. Mirón-Canelo JA, Iglesias-De Sena H, Alonso-Sardón M. Valoración de los estudiantes sobre su formación en la facultad de medicina. Educ Med 2011; 14: 221-8. Disponible en: http://scielo.isciii.es/ scielo.php?script=sci_abstract\&pid=S1575-18132011000400005

25. Castillo-Tarrillo GF, Vetanzo-Sánchez G, Caballero-Ortiz AG. Formación integral en las facultades de medicina de Perú: objetivo más allá del examen nacional. Rev la Fund Educ Médica. 2016;19(6):311. Disponible en: http://scielo.isciii.es/scielo. php?script=sci_arttext\&pid=S2014-98322016000600007\&lng=es.

26. Gutiérrez SM, Llosa IMPL. El programa de medicina en la Universidad Peruana Cayetano Heredia. Rev Peru Med Exp Salud Publica. 2014;31:582-7. Disponible en: http://www.scielo.org.pe/scielo. php?script=sci_abstract \&pid=S1726-46342014000300025\&lng= es\&nrm=iso

27. Huamaní C, Gutiérrez C, Mezones-holguín E. Correlación y concordancia entre el Examen Nacional de Medicina y el promedio ponderado universitario: análisis de la experiencia peruana en el periodo 2007-2009. Rev Peru Med Exp Salud Publica. 2011;28:6271. Disponible en: http://www.scielo.org.pe/scielo.php?script=sci_ arttext\&pid=S1726-46342011000100010

28. Costa-Santos C, Vieira-Marques $P$, Costa-Pereira A, Ferreira MA, Freitas A. Do students from public schools fare better in medical school than their colleagues from private schools? if so, what can we learn from this? BMC Med Educ. 2018;18(1). doi: 10.1186/ s12909-018-1162-x

29. Hernández-Gálvez DC, Roldán-Valadez E. Mexican ENARM: Performance comparison of public vs. private medical schools, geographic and socioeconomic regions. Salud Publica Mex. 2019; 61(5):637-47. doi: 10.21149/10078 